\title{
Hu Moment Invariant: A New Method for Hearing Loss Detection
}

\author{
Lijun Tang ${ }^{1}$, Yixuan Qi ${ }^{1, *}$ and Atiena Pereira ${ }^{2}$ \\ ${ }^{1}$ School of Computer Science and Engineering, Nanjing Normal University, Nanjing 210023, P R \\ China \\ ${ }^{2}$ School of Biology, University of Campinas, Campinas, São Paulo, Brazil \\ lijun-tang@qq.com, process_ing@qq.com, apereira@ieee.org
}

Keywords: Hu moment invariant; Support vector machine; Hearing loss; Detection; Identification.

\begin{abstract}
This paper proposed a novel hearing loss detection method. Our method first used seven Hu moment invariants to extract features. Afterwards, we used support vector machine to act as the classifier. The 10x5-fold cross validation shows our method yielded an overall accuracy of $77.47 \pm$ $1.17 \%$. The sensitivities of healthy control, left-sided hearing loss, and right-sided hearing loss are $77.60 \pm 5.72 \%, 77.60 \pm 4.30 \%$, and $77.20 \pm 5.98 \%$, respectively. In all, our method is effective in hearing loss identification.
\end{abstract}

\section{Introduction}

Hearing loss (HL) [1] is a type of hearing impairment. It may be caused by ageing, noise, infection, genetics, etc. Hence, testing for poor hearing is recommended for all new-born babies. Nevertheless, more than half of hearing loss is preventable [2]. Therefore, it is important to early detect hearing loss, and then give patients proper treatments.

Currently, scholars proposed several feasible approaches. Li [3] proposed a fractional Fourier transform method. Jia [4] used deep autoencoder method. Wang [5] suggested to use dual-tree complex wavelet transform. Li [6] offered a new method using fitness-scaling adaptive genetic algorithm. Nayak [7] used stationary wavelet transform and Shannon entropy. Chen [8] gave a new method of using generalized eigenvalue proximal support vector machine. Gorriz [9] employed directed acyclic graph support vector machine. Except above literature, image processing [10, 11] techniques and artificial intelligence $[12,13]$ methods were already applied to medical image processing.

Nevertheless, those methods are too complicated and time-consuming. In this study, we proposed a novel method that combined Hu moment invariant (HMI) and support vector machine. Our method also achieves good results for hearing loss identification.

\section{Materials}

We collected 75 magnetic resonance brain images from local hospitals. The dataset can be divided into three categories, including 25 healthy images, 25 left deafness images, and 25 right deafness images. Written consents were obtained from all subjects. Different types of hearing loss images are shown below in Figure. 1

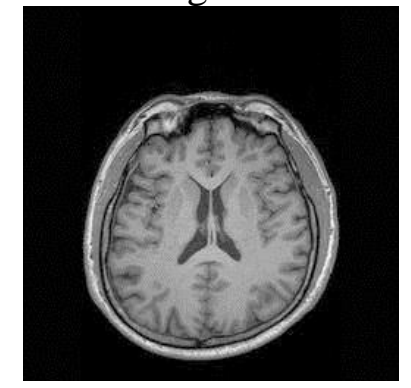

(a) healthy control

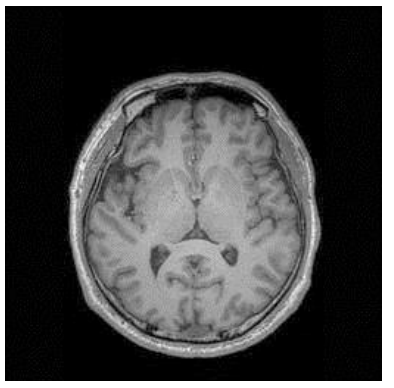

(b) left-sided hearing loss

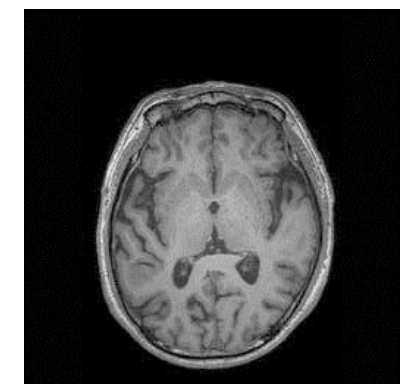

(c) right-sided hearing loss

Figure 1. Sample of brain images 


\section{Hu Moment Invariant}

We used the image moment [14] as the shape descriptor. For a 2D healthy or hearing loss brain image $F(x, y)$, we can define the raw moment $R$ of order $(b+d)$ as

$$
R_{b d}=\sum_{x} \sum_{y} x^{b} \times y^{d} \times F(x, y)
$$

where $b, d=0,1,2, \ldots$ The central moments $\mu$ replaced the raw moment $R$ in formula.(1)

$$
\begin{gathered}
\mu_{b d}=\sum_{x} \sum_{y}(x-\bar{x})^{b} \times(y-\bar{y})^{d} \times F(x, y) \\
\bar{x}=\frac{R_{10}}{R_{00}}, \bar{y}=\frac{R_{01}}{R_{00}}
\end{gathered}
$$

Central moments are translational-invariant [15]. It can be extended to be both translation- and scale- invariant [16]. The new moment was called normalized central moment $Q$.

$$
Q_{b d}=\frac{\mu_{b d}}{\mu_{00}^{\left(\frac{b+d}{2}+1\right)}}
$$

To enable invariance to rotation, $\mathrm{Hu}[17]$ proposed seven moments $P$ as

$$
\begin{gathered}
P_{1}=Q_{20}+Q_{02} \\
P_{2}=\left(Q_{20}-\eta_{02}\right)^{2}+Q \eta_{11}^{2} \\
P_{3}=\left(Q_{30}-3 Q_{12}\right)^{2}+\left(3 Q_{21}-Q_{03}\right)^{2} \\
P_{4}=\left(Q_{30}+Q_{12}\right)^{2}+\left(Q_{21}+Q_{03}\right)^{2} \\
P_{5}=\left(Q_{30}-3 Q_{12}\right)\left(Q_{30}+Q_{12}\right)\left[\left(Q_{30}+Q_{12}\right)^{2}-3\left(Q_{21}+Q_{03}\right)^{2}\right]+ \\
\left(3 Q_{21}-Q_{03}\right)\left(Q_{21}+Q_{03}\right)\left[3\left(Q_{30}+Q_{12}\right)^{2}-\left(Q_{21}+Q_{03}\right)^{2}\right] \\
P_{6}=\left(Q_{20}-Q_{02}\right)\left[\left(Q_{30}+Q_{12}\right)^{2}-\left(Q_{21}+Q_{03}\right)^{2}\right]+4 Q_{11}\left(Q_{30}+Q_{12}\right)\left(Q_{21}+Q_{03}\right) \\
P_{7}=\left(3 Q_{21}-Q_{03}\right)\left(Q_{30}+Q_{12}\right)\left[\left(Q_{30}+Q_{12}\right)^{2}-3\left(Q_{21}+Q_{03}\right)^{2}\right]- \\
\left(Q_{30}-3 Q_{12}\right)\left(Q_{21}+Q_{03}\right)\left[3\left(Q_{30}+Q_{12}\right)^{2}-\left(Q_{21}+Q_{03}\right)^{2}\right]
\end{gathered}
$$

Hu moment invariants $P=\left[P_{1}, P_{2}, \ldots, P_{7}\right]$ are a set of absolute orthogonal (i.e. rotation) moment invariants. Afterwards, we used support vector machine (SVM) [18-21] as the classifier. The optimal parameters of SVM were obtained by grid-searching method [22-25]. The 10x5-fold cross validation was employed. We did not use deep learning techniques [26-28], since our dataset is small and deep learning does not perform well on small-size dataset.

\section{Experiments and Results}

The statistical results are obtained and listed below in Table. Each fold contains five healthy controls, five left-sided hearing loss brains, and five right-sided hearing loss brains. The element in Table of $(a+b+c)=d$ means $a$ healthy controls, $b$ left-sided, and $c$ right-sided were correctly identified. In total $d$ brains were identified.

Table 1 Correctly identified results

\begin{tabular}{|l|l|l|l|l|l|l|}
\hline & $F 1$ & $F 2$ & $F 3$ & $F 4$ & F5 & Total \\
\hline R1 & $5+3+3=11$ & $2+4+4=10$ & $5+4+3=12$ & $4+3+5=12$ & $5+5+3=13$ & $21+19+18=58$ \\
\hline R2 & $3+3+4=10$ & $4+4+4=12$ & $5+4+4=13$ & $4+4+4=12$ & $4+3+5=12$ & $20+18+21=59$ \\
\hline R3 & $4+4+4=12$ & $4+3+4=11$ & $4+5+4=13$ & $4+5+3=12$ & $4+4+3=11$ & $20+21+18=59$ \\
\hline R4 & $2+4+5=11$ & $4+4+3=11$ & $5+4+4=13$ & $3+4+5=12$ & $3+4+3=10$ & $17+20+20=57$ \\
\hline R5 & $3+4+3=10$ & $3+3+5=11$ & $3+4+4=11$ & $4+4+4=12$ & $4+4+5=13$ & $17+19+21=57$ \\
\hline R6 & $3+3+3=9$ & $4+4+4=12$ & $5+4+4=13$ & $3+4+5=12$ & $4+4+4=12$ & $19+19+20=58$ \\
\hline R7 & $4+5+4=13$ & $5+2+4=11$ & $3+5+3=11$ & $4+4+4=12$ & $4+3+4=11$ & $20+19+19=58$ \\
\hline R8 & $4+5+4=13$ & $4+4+5=13$ & $4+4+4=12$ & $3+4+2=9$ & $4+4+2=10$ & $19+21+17=57$ \\
\hline R9 & $3+4+5=12$ & $4+2+5=11$ & $5+4+4=13$ & $3+4+2=9$ & $5+4+5=14$ & $20+18+21=59$ \\
\hline R10 & $4+4+4=12$ & $3+5+3=11$ & $5+3+3=11$ & $5+4+4=13$ & $4+4+4=12$ & $21+20+18=59$ \\
\hline
\end{tabular}


The total confusion matrix of 10 runs was listed in Table 2. Here $\mathrm{C} 1$ represents healthy control, $\mathrm{C} 2$ represents left-sided hearing loss, and $\mathrm{C} 3$ represents right-sided hearing loss. The sensitivities of each class are listed in Table 3. The average sensitivities of three classes are $77.60 \pm 5.72 \%, 77.60 \pm$ $4.30 \%$, and $77.20 \pm 5.98 \%$. The overall accuracies of the three classes over 10 runs are listed in Table 4. In average, the overall accuracy is $77.47 \pm 1.17 \%$.

Table 2 Confusion matrix

\begin{tabular}{|l|l|l|l|}
\hline & C1 & C2 & C3 \\
\hline C1 & 194 & 27 & 29 \\
\hline C2 & 31 & 194 & 25 \\
\hline C3 & 26 & 31 & 193 \\
\hline
\end{tabular}

Table 3 Sensitivity of three classes of 10 runs

\begin{tabular}{|l|l|l|l|l|l|l|l|l|l|l|l|l|l|l|l|l|l|l|}
\hline \multicolumn{1}{|c|}{ Healthy control } & \multicolumn{4}{c|}{ Left-sided hearing loss } & \multicolumn{5}{|c|}{ Right-sided hearing loss } \\
\hline & F1 & F2 & F3 & F4 & F5 & T & F1 & F2 & F3 & F4 & F5 & T & F1 & F2 & F3 & F4 & F5 & T \\
\hline R1 & 100 & 40 & 100 & 80 & 100 & 84 & 60 & 80 & 80 & 60 & 100 & 76 & 60 & 80 & 60 & 100 & 60 & 72 \\
\hline R2 & 60 & 80 & 100 & 80 & 80 & 80 & 60 & 80 & 80 & 80 & 60 & 72 & 80 & 80 & 80 & 80 & 100 & 84 \\
\hline R3 & 80 & 80 & 80 & 80 & 80 & 80 & 80 & 60 & 100 & 100 & 80 & 84 & 80 & 80 & 80 & 60 & 60 & 72 \\
\hline R4 & 40 & 80 & 100 & 60 & 60 & 68 & 80 & 80 & 80 & 80 & 80 & 80 & 100 & 60 & 80 & 100 & 60 & 80 \\
\hline R5 & 60 & 60 & 60 & 80 & 80 & 68 & 80 & 60 & 80 & 80 & 80 & 76 & 60 & 100 & 80 & 80 & 100 & 84 \\
\hline R6 & 60 & 80 & 100 & 60 & 80 & 76 & 60 & 80 & 80 & 80 & 80 & 76 & 60 & 80 & 80 & 100 & 80 & 80 \\
\hline R7 & 80 & 100 & 60 & 80 & 80 & 80 & 100 & 40 & 100 & 80 & 60 & 76 & 80 & 80 & 60 & 80 & 80 & 76 \\
\hline R8 & 80 & 80 & 80 & 60 & 80 & 76 & 100 & 80 & 80 & 80 & 80 & 84 & 80 & 100 & 80 & 40 & 40 & 68 \\
\hline R9 & 60 & 80 & 100 & 60 & 100 & 80 & 80 & 40 & 80 & 80 & 80 & 72 & 100 & 100 & 80 & 40 & 100 & 84 \\
\hline R10 & 80 & 60 & 100 & 100 & 80 & 84 & 80 & 100 & 60 & 80 & 80 & 80 & 80 & 60 & 60 & 80 & 80 & 72 \\
\hline
\end{tabular}

Table 4 Accuracy of three classes of 10 runs

\begin{tabular}{|l|l|l|l|l|l|l|}
\hline & F1 & F2 & F3 & F4 & F5 & Total \\
\hline R1 & 73.33 & 66.67 & 80.00 & 80.00 & 86.67 & 77.33 \\
\hline R2 & 66.67 & 80.00 & 86.67 & 80.00 & 80.00 & 78.67 \\
\hline R3 & 80.00 & 73.33 & 86.67 & 80.00 & 73.33 & 78.67 \\
\hline R4 & 73.33 & 73.33 & 86.67 & 80.00 & 66.67 & 76.00 \\
\hline R5 & 66.67 & 73.33 & 73.33 & 80.00 & 86.67 & 76.00 \\
\hline R6 & 60.00 & 80.00 & 86.67 & 80.00 & 80.00 & 77.33 \\
\hline R7 & 86.67 & 73.33 & 73.33 & 80.00 & 73.33 & 77.33 \\
\hline R8 & 86.67 & 86.67 & 80.00 & 60.00 & 66.67 & 76.00 \\
\hline R9 & 80.00 & 73.33 & 86.67 & 60.00 & 93.33 & 78.67 \\
\hline R10 & 80.00 & 73.33 & 73.33 & 86.67 & 80.00 & 78.67 \\
\hline
\end{tabular}

\section{Conclusion}

This study introduced to use $\mathrm{Hu}$ moment invariant, and proved its effectiveness in hearing loss detection. The future work is to test other manual features, and try to enroll more students to enlarge our dataset. In addition, transfer learning [29] technique and fractal [30] will be tested. Besides, our method can be applied to detect abnormal breast [31-33] and other diseases.

\section{Reference}

[1] Sanyelbhaa, H., A. Kabel, et al., The risk ratio for development of hereditary sensorineural hearing loss in consanguineous marriage offspring. International Journal of Pediatric Otorhinolaryngology, 2017. 101: pp. 7-10 
[2] Muus, J.S., F.W. Weir, et al., Hearing loss in children with growth hormone deficiency. International Journal of Pediatric Otorhinolaryngology, 2017. 100: pp. 107-113

[3] Li, J., Detection of Left-Sided and Right-Sided Hearing Loss via Fractional Fourier Transform. Entropy, 2016. 18(5), Article ID: 194

[4] Jia, W., Three-Category Classification of Magnetic Resonance Hearing Loss Images Based on Deep Autoencoder. Journal of Medical Systems, 2017. 41, Article ID: 165

[5] Wang, S., Y. Zhang, et al., Preliminary Study on Unilateral Sensorineural Hearing Loss Identification via Dual-Tree Complex Wavelet Transform and Multinomial Logistic Regression, in Natural and Artificial Computation for Biomedicine and Neuroscience: International Work-Conference on the Interplay Between Natural and Artificial Computation, IWINAC 2017, Corunna, Spain, June 19-23, 2017, Proceedings, Part I, eds. J.M. Ferrández Vicente, et al., 2017, Springer International Publishing: Cham. pp. 289-297.

[6] Li, J., Texture analysis method based on fractional Fourier entropy and fitness-scaling adaptive genetic algorithm for detecting left-sided and right-sided sensorineural hearing loss. Fundamenta Informaticae, 2017. 151(1-4): pp. 505-521

[7] Nayak, D.R., Detection of unilateral hearing loss by Stationary Wavelet Entropy. CNS \& Neurological Disorders - Drug Targets, 2017. 16(2): pp. 122-128

[8] Chen, Y. and X.-Q. Chen Sensorineural hearing loss detection via discrete wavelet transform and principal component analysis combined with generalized eigenvalue proximal support vector machine and Tikhonov regularization. Multimedia Tools and Applications, 2016, DOI: 10.1007/s11042-016-4087-6 (Online).

[9] Gorriz, J.M. and J. Ramírez, Wavelet entropy and directed acyclic graph support vector machine for detection of patients with unilateral hearing loss in MRI scanning. Frontiers in Computational Neuroscience, 2016. 10, Article ID: 160

[10] Wei, G., Color Image Enhancement based on HVS and PCNN. SCIENCE CHINA Information Sciences, 2010. 53(10): pp. 1963-1976

[11] Wu, L.N., Improved image filter based on SPCNN. Science In China Series F-Information Sciences, 2008. 51(12): pp. 2115-2125

[12]Zhan, T., Pathological brain detection by artificial intelligence in magnetic resonance imaging scanning. Progress in Electromagnetics Research, 2016. 156: pp. 105-133

[13] Ji, G., A comprehensive survey on particle swarm optimization algorithm and its applications. Mathematical Problems in Engineering, 2015. 2015, Article ID: 931256

[14] Li, B.Q., S.H. Lu, et al., Tchebichef-Hermite image moment method: A novel tool for chemometric analysis of three-dimensional spectra. Chemometrics and Intelligent Laboratory Systems, 2017. 167: pp. 36-43

[15] Karakasis, E.G., A. Amanatiadis, et al., Image moment invariants as local features for content based image retrieval using the Bag-of-Visual-Words model. Pattern Recognition Letters, 2015. 55: pp. 22-27

[16] Yang, J., Pathological brain detection in MRI scanning via Hu moment invariants and machine learning. Journal of Experimental \& Theoretical Artificial Intelligence, 2017. 29(2): pp. 299-312

[17] Hu, M.-K., Visual pattern recognition by moment invariants. IRE Transactions on Information Theory, 1962. 8(2): pp. 179-187 
[18] Wu, L., An MR brain images classifier via principal component analysis and kernel support vector machine. Progress In Electromagnetics Research, 2012. 130: pp. 369-388

[19] Wu, L., An MR Brain Images Classifier via Principal Component Analysis and Kernel Support Vector Machine. Progress in Electromagnetics Research, 2012. 130(1): pp. 369-388

[20]Li, Y., Detection of Dendritic Spines using Wavelet Packet Entropy and Fuzzy Support Vector Machine. CNS \& Neurological Disorders - Drug Targets, 2017. 16(2): pp. 116-121

[21]Lu, H.M., Facial Emotion Recognition Based on Biorthogonal Wavelet Entropy, Fuzzy Support Vector Machine, and Stratified Cross Validation. IEEE Access, 2016. 4: pp. $8375-8385$

[22] Liu, A., Magnetic resonance brain image classification via stationary wavelet transform and generalized eigenvalue proximal support vector machine. Journal of Medical Imaging and Health Informatics, 2015. 5(7): pp. 1395-1403

[23]Ji, G., An MR brain images classifier system via particle swarm optimization and kernel support vector machine. The Scientific World Journal, 2013, Article ID: 130134

[24] Dong, Z., Classification of Alzheimer disease based on structural magnetic resonance imaging by kernel support vector machine decision tree. Progress In Electromagnetics Research, 2014. 144: pp. 171-184

[25] Yang, J., Preclinical diagnosis of magnetic resonance (MR) brain images via discrete wavelet packet transform with Tsallis entropy and generalized eigenvalue proximal support vector machine (GEPSVM). Entropy, 2015. 17(4): pp. 1795-1813

[26] Jia, W. Five-category classification of pathological brain images based on deep stacked sparse autoencoder. Multimedia Tools and Applications, 2017, DOI: 10.1007/s11042-017-5174-Z (Online).

[27] Sun, J. Polarimetric synthetic aperture radar image segmentation by convolutional neural network using graphical processing units. Journal of Real-Time Image Processing, 2017, DOI: 10.1007/s11554-017-0717-0 (Online).

[28] Jiang, Y., X. Hou, et al., Cerebral Micro-Bleed Detection Based on the Convolution Neural Network With Rank Based Average Pooling. IEEE Access, 2017. 5: pp. 16576-16583

[29] Salaken, S.M., A. Khosravi, et al., Extreme learning machine based transfer learning algorithms: A survey. Neurocomputing, 2017. 267: pp. 516-524

[30]ZHANG, Y., Synthetic Minority Oversampling Technique and Fractal Dimension for Identifying Multiple Sclerosis. Fractals, 2017. 25(4), Article ID: 1740010

[31]Liu, G., Computer-aided diagnosis of abnormal breasts in mammogram images by weighted-type fractional Fourier transform. Advances in Mechanical Engineering, 2016. 8(2), Article ID: 11

[32] Rao, R.V., A. Liu, et al., Abnormal Breast Detection in Mammogram Images by Feed-forward Neural Network trained by Jaya Algorithm. Fundamenta Informaticae, 2017. 151(1-4): pp. 191-211

[33] Wu, X., Smart detection on abnormal breasts in digital mammography based on contrast-limited adaptive histogram equalization and chaotic adaptive real-coded biogeography-based optimization. Simulation, 2016. 92(9): pp. 873-885 